University of Windsor

Scholarship at UWindsor

1990

\title{
An alternative proof of some relations between hydrogenic matrix elements
}

\author{
R. A. Swainson \\ Gordon W. F. Drake \\ University of Windsor
}

Follow this and additional works at: https://scholar.uwindsor.ca/physicspub

Part of the Physics Commons

\section{Recommended Citation}

Swainson, R. A. and Drake, Gordon W. F.. (1990). An alternative proof of some relations between hydrogenic matrix elements. Journal of Physics B: Atomic, Molecular and Optical Physics, 23 (7), 1079-1084.

https://scholar.uwindsor.ca/physicspub/57

This Article is brought to you for free and open access by the Department of Physics at Scholarship at UWindsor. It has been accepted for inclusion in Physics Publications by an authorized administrator of Scholarship at UWindsor. For more information, please contact scholarship@uwindsor.ca. 
An alternative proof of some relations between hydrogenic matrix elements

This article has been downloaded from IOPscience. Please scroll down to see the full text article.

1990 J. Phys. B: At. Mol. Opt. Phys. 231079

(http://iopscience.iop.org/0953-4075/23/7/005)

View the table of contents for this issue, or go to the journal homepage for more

Download details:

IP Address: 137.207.184.30

The article was downloaded on 08/05/2013 at 19:42

Please note that terms and conditions apply. 


\title{
An alternative proof of some relations between hydrogenic matrix elements
}

\author{
Robin A Swainson and G W F Drake \\ Department of Physics, University of Windsor, Windsor, Ontario, Canada N9B 3P4
}

Received 27 October 1989

\begin{abstract}
A relationship between certain integrals of the product of two Laguerre polynomials is obtained. It is shown how this relationship, together with two known Hankel transforms, allows for an alternative derivation of some relations between matrix elements of $r^{k}$ for hydrogenic ions. A further relation, involving matrix elements of logarithmic functions, is obtained in a similar manner.
\end{abstract}

\section{Introduction}

Some years ago Blanchard [1] discovered a recurrence relation between matrix elements of hydrogenic radial wavefunctions from which he was able to deduce that, for $\left|l-l^{\prime}\right| \leqslant k \leqslant l+l^{\prime}$

$$
\begin{aligned}
\left\langle n l^{\prime}\left|r^{-k-2}\right| n l\right\rangle= & (-1)^{l-l^{\prime}}(2 / a n)^{2 k+1}\left(l+l^{\prime}-k\right) ! k !^{2} \\
& \times\left\langle n l^{\prime}\left|r^{k-1}\right| n l\right\rangle /\left[\left(l+l^{\prime}+1+k\right) !\left(k+l-l^{\prime}\right) !\left(k+l^{\prime}-l\right) !\right] .
\end{aligned}
$$

The matrix elements are defined such that, given any function $h(r)$,

$$
\left\langle n^{\prime} l^{\prime}|h(r)| n l\right\rangle=\int_{0}^{\infty} R_{n^{\prime} r^{\prime}}(r) h(r) R_{n l}(r) r^{2} \mathrm{~d} r
$$

where the radial functions are [2]

$$
\begin{aligned}
& R_{n l}(r)=N_{n l} r^{l} \mathrm{e}^{-r / a n} L_{n-l-1}^{2 l+1}(2 r / a n) \\
& N_{n l}=\left(2 / n^{2} a^{3 / 2}\right)[(n-l-1) ! /(n+l) !]^{1 / 2}(2 / a n)^{l}
\end{aligned}
$$

and $a=a_{0} / Z$ is the scaled Bohr radius. $L_{n}^{\alpha}(x)$ is a generalised Laguerre polynomial [3].

The identity (1.1) generalised two earlier results by Pasternak [4] and Pasternak and Sternheimer [5]. Pasternak [4] showed that, for $k=0,1, \ldots, 2 l$

$$
\left\langle n l\left|r^{-k-2}\right| n l\right\rangle=(2 / a n)^{2 k+1}(2 l-k) ! /(2 l+1+k) !\left\langle n l\left|r^{k-1}\right| n l\right\rangle
$$

while Pasternak and Sternheimer [5] showed that, for $l \neq l^{\prime}$ and $k=0,1, \ldots,\left|l-l^{\prime}\right|-1$

$$
\left\langle n l^{\prime}\left|r^{-k-2}\right| n l\right\rangle=0
$$

The significance of these identities is that they relate the radial matrix elements of positive and negative powers of $r$ (although the matrix element of $r^{-1}$ is not covered).

The relation (1.1) was rediscovered (though not proven) by More [6], and a further proof, relying on various properties of generalised hypergeometric functions of unit 
argument, was found by Ojha and Crothers [7]. Several group-theoretical investigations of hydrogenic matrix elements have been published, notably that by Armstrong [8], wherein the properties of a particular physical realisation of the $\mathrm{SO}(2,1)$ Lie algebra are shown to lead to the Pasternak-Sternheimer identity (1.6).

The analysis of hydrogenic radial matrix elements continues to attract the attention of workers in various branches of atomic physics. The Schrödinger radial ladder operator [9] has been found to lead to recurrence relations between such matrix elements, including some which are special cases of (1.1). In the context of manyelectron theory hydrogenic radial integrals have been found to be useful for calculations employing the screened hydrogenic model [10]. And the long-standing problem of deriving tight closed-form expressions for the radial matrix elements remains essentially unsolved (see though [11] and [12] for special cases).

A further proof of (1.1), though of interest in and of itself, is useful in view of the light it may shed on the underlying principles behind that and similar results. We have found a curious relationship (2.9) between certain integrals of products of two Laguerre polynomials which, together with well established Hankel transformations, leads us to a proof of (1.1) entirely within the framework of classical special function theory. This work will be presented in section 2 . We have been unable to find any previous reference to the relationship given in (2.9). Our work is in some ways complementary to that of Ojha and Crothers [7] in that we have dissected their direct proof into several distinct steps which highlight the few specific properties of Laguerre polynomials which lead to (1.1). Nonetheless, our proof, which was found independently, is somewhat more general. All three relations, $(1.1),(1.5)$ and (1.6) follow from our analysis.

Blanchard [1] also discovered a relationship between matrix elements of $\log r$ and powers of $r$. In section 3 we extend our work to include logarithmic factors in the integrands and show how, in much the same manner, we can derive Blanchard's additional formulae. Such an extension to the logarithmic case, though relatively straightforward in the work presented here, was not attempted in [7].

\section{Matrix elements of $r^{k}$}

The following generating function for Laguerre polynomials [13],

$$
\sum_{i=0}^{\infty} t^{i} L_{i}^{\alpha-i}(x)=(1+t)^{\alpha} \mathrm{e}^{-t x}
$$

implies that

$$
\begin{aligned}
\sum_{p, q} t^{p} s^{q} \int_{0}^{\infty} \mathrm{e}^{-x} x^{\gamma} L_{p}^{\beta-p+m}(x) L_{q}^{\alpha-q+n}(x) \mathrm{d} x \\
=\Gamma(\gamma+1)(1+t)^{\beta+m}(1+s)^{\alpha+n} /(1+t+s)^{\gamma+1}
\end{aligned}
$$

from which we can deduce that

$$
\begin{aligned}
& \int_{0}^{\infty} \mathrm{e}^{-x} x^{\gamma} L_{n}^{\beta-n+m}(x) L_{m}^{\alpha-m+n}(x) \mathrm{d} x \\
& \quad=\left.\frac{\Gamma(\gamma+1)}{m ! n !} \frac{\partial^{n}}{\partial t^{n}} \frac{\partial^{m}}{\partial s^{m}}\left[(1+t)^{\beta+m}(1+s)^{\alpha+n} /(1+t+s)^{\gamma+1}\right]\right|_{t=s=0} .
\end{aligned}
$$


Since

$$
[(1+t)(1+s) /(1+t+s)]^{\gamma+1}=\sum_{i=0}^{\infty}(\gamma+1)_{i} / i ![t s /(1+t)(1+s)]^{i}
$$

and

$$
\left.\frac{\mathrm{d}^{p}}{\mathrm{~d} x^{p}} x^{i}(1+x)^{-\sigma-i}\right|_{x=0}=(-1)^{p}(-p)_{i}(\sigma)_{p} /(\sigma)_{i}
$$

it follows that

$$
\begin{aligned}
\int_{0}^{\infty} \mathrm{e}^{-x} x^{\gamma} L_{n}^{\beta-n+m}(x) L_{m}^{\alpha-m+n}(x) \mathrm{d} x \\
={ }_{3} F_{2}\left[\begin{array}{c}
-n,-m, \gamma+1 ; 1 \\
\gamma+1-m-\beta, \gamma+1-n-\alpha
\end{array}\right](-1)^{n+m} \Gamma(\gamma+1) \\
\quad \times(\gamma+1-m-\beta)_{n}(\gamma+1-n-\alpha)_{m} /(n ! m !)
\end{aligned}
$$

where the generalised hypergeometric function of unit argument is defined in the usual way [13] and $(\sigma)_{i}=\Gamma(\sigma+i) / \Gamma(\sigma)$.

Letting $\alpha \rightarrow \beta-n+m$ and $\beta \rightarrow \alpha-m+n$ leads immediately to

$$
\begin{aligned}
\int_{0}^{\infty} \mathrm{e}^{-x} x^{\gamma} L_{n}^{\alpha}(x) & L_{m}^{\beta}(x) \mathrm{d} x \\
= & { }_{3} F_{2}\left[\begin{array}{c}
-n,-m, \gamma+1 ; 1 \\
\gamma-\alpha-n+1, \gamma-\beta-m+1
\end{array}\right](-1)^{m+n} \Gamma(\gamma+1) \\
& \times(\gamma+1-\alpha-n)_{n}(\gamma+1-\beta-m)_{m} /(n ! m !) .
\end{aligned}
$$

It is not difficult to see that if $\gamma-\alpha=\gamma-\beta=k$, a non-negative integer less than $|m-n|$, then the integral vanishes:

$$
\int_{0}^{\infty} \mathrm{e}^{-x} x^{\gamma} L_{n}^{\gamma-k}(x) L_{m}^{\gamma-k}(x) \mathrm{d} x=0 \quad k=0,1, \ldots,|m-n|-1 .
$$

Finally, since $(\sigma-n)_{m} /(\sigma-m)_{n}=(\sigma)_{m-n},(2.6)$ and (2.7) together imply that

$$
\begin{aligned}
\int_{0}^{\infty} \mathrm{e}^{-x} x^{\gamma} L_{n}^{\alpha}(x) L_{m}^{\beta}(x) \mathrm{d} x \\
=\int_{0}^{\infty} \mathrm{e}^{-x} x^{\gamma} L_{n}^{\beta-n+m}(x) L_{m}^{\alpha-m+n}(x) \mathrm{d} x \\
\quad \times\left[(\gamma-\alpha+1)_{m-n}(\gamma-\beta+1)_{n-m}\right]^{-1} .
\end{aligned}
$$

The formulae given in (2.8) and (2.9) constitute the main result of this paper.

Specific choices of the parameters in (2.8) and (2.9) give rise to several interesting results. We make mention of only one, since it constitutes a sort of generalised orthogonality property of Laguerre polynomials. Put $\alpha=\gamma-k$ in (2.8); then

$$
\int_{0}^{\infty} \mathrm{e}^{-x} x^{\alpha+k} L_{n}^{\alpha}(x) L_{m}^{\alpha}(x) \mathrm{d} x=0 \quad k=0,1, \ldots,|m-n|-1
$$

which, when $k=0$ and $n \neq m$, reduces to the usual orthogonality condition [3]. 
Another specific choice of the parameters leads to a proof of (1.1). Erdelyi [14] has shown that

$\mathrm{e}^{-x} x^{\sigma} L_{p}^{\sigma+q-p}(x) L_{q}^{\sigma+p-q}(x)=(-1)^{p+q} \int_{0}^{\infty} \mathrm{e}^{-y} y^{\sigma} L_{p}^{\sigma}(y) L_{q}^{\sigma}(y) J_{2 \sigma}(2 \sqrt{x y}) \mathrm{d} y$

where $J_{\nu}$ is a Bessel function of order $\nu$, and since [13]

$\int_{0}^{\infty} x^{-\kappa-1} J_{2 \sigma}(2 \sqrt{x y}) \mathrm{d} x=y^{\kappa} \Gamma(\sigma-\kappa) / \Gamma(\sigma+\kappa+1) \quad(0<\kappa<\sigma)$

we can deduce that

$$
\begin{aligned}
\int_{0}^{\infty} \mathrm{e}^{-x} x^{\sigma-\kappa-1} L_{p}^{\sigma+q-p}(x) L_{q}^{\sigma+p-q}(x) \mathrm{d} x \\
=(-1)^{p+q} \int_{0}^{\infty} x^{-\kappa-1} \int_{0}^{\infty} \mathrm{e}^{-y} y^{\sigma} L_{p}^{\sigma}(y) L_{q}^{\sigma}(y) J_{2 \sigma}(2 \sqrt{x y}) \mathrm{d} y \mathrm{~d} x \\
=(-1)^{p+q} \int_{0}^{\infty} \mathrm{e}^{-y} y^{\sigma} L_{p}^{\sigma}(y) L_{q}^{\sigma}(y) \int_{0}^{\infty} x^{-\kappa-1} J_{2 \sigma}(2 \sqrt{x y}) \mathrm{d} x \mathrm{~d} y \\
=(-1)^{p+q} \Gamma(\sigma-\kappa) / \Gamma(\sigma+\kappa+1) \int_{0}^{\infty} \mathrm{e}^{-y} y^{\sigma+\kappa} L_{p}^{\sigma}(y) L_{q}^{\sigma}(y) \mathrm{d} y .
\end{aligned}
$$

Finally, employing our integral formulae (2.8) and (2.9), we have

$$
\begin{aligned}
\int_{0}^{\infty} \mathrm{e}^{-x} x^{\sigma-\kappa-1} & L_{p}^{\sigma+q-p}(x) L_{q}^{\sigma+p-q}(x) \mathrm{d} x \\
= & (-1)^{p+q} \Gamma(\sigma-\kappa) /\left[\Gamma(\sigma+\kappa+1)(\kappa+1)_{p-q}(\kappa+1)_{q-p}\right] \\
& \times \int_{0}^{\infty} \mathrm{e}^{-y} y^{\sigma+\kappa} L_{p}^{\sigma+q-p}(y) L_{q}^{\sigma+p-q}(y) \mathrm{d} y
\end{aligned}
$$

for $0<\kappa<\sigma$ with the integral vanishing for $\kappa$ a positive integer less than $|p-q|$.

We now have everything necessary to prove the three identities given in the introduction. Letting $p=n-l-1, q=n-l^{\prime}-1$, and $\sigma=l+l^{\prime}+1$ and noting the definition of the radial hydrogenic wavefunctions (1.3) we see that (2.14) implies

$$
\left\langle n l^{\prime}\left|r^{-\kappa-2}\right| n l\right\rangle=0
$$

for $\kappa$ a non-negative integer less than $\left|l-l^{\prime}\right|$, and

$$
\begin{aligned}
\left\langle n l^{\prime}\left|r^{-\kappa-2}\right| n l\right\rangle= & (-1)^{l+l^{\prime}} \frac{\Gamma\left(l+l^{\prime}-\kappa+1\right) \Gamma(\kappa+1)^{2}}{\Gamma\left(l+l^{\prime}+\kappa+2\right) \Gamma\left(\kappa+l-l^{\prime}+1\right) \Gamma\left(\kappa+l^{\prime}-l+1\right)} \\
& \times(2 / a n)^{2 \kappa+1}\left\langle n l^{\prime}\left|r^{\kappa-1}\right| n l\right\rangle
\end{aligned}
$$

for all other $\kappa$ such that $0 \leqslant \kappa \leqslant l+l^{\prime}$.

\section{Matrix elements of $r^{k} \log r$}

The matrix elements of $\log r$ can be treated in a manner entirely similar to those of $r$. To achieve this we first derive an anologue of our integral formula (2.9). Noting that

$$
x^{\gamma} \log x=\mathrm{d} / \mathrm{d} \gamma\left(x^{\gamma}\right)
$$


and [3]

$$
\mathrm{d} / \mathrm{d} \sigma \Gamma(\sigma)=\Gamma(\sigma) \psi(\sigma)
$$

on differentiating (2.9) with respect to $\gamma$ we find

$$
\begin{aligned}
\int_{0}^{\infty} \mathrm{e}^{-x} x^{\gamma}[\log & x-\psi(\gamma+1-\alpha)-\psi(\gamma+1-\beta)] L_{n}^{\alpha}(x) L_{m}^{\beta}(x) \mathrm{d} x \\
= & \int_{0}^{\infty} \mathrm{e}^{-x} x^{\gamma}[\log x-\psi(\gamma+1-\alpha+m-n)-\psi(\gamma+1-\beta+n-m)] \\
& \times L_{n}^{\beta-n+m}(x) L_{m}^{\alpha-m+n}(x) \mathrm{d} x /(\gamma-\alpha+1)_{m-n}(\gamma-\beta+1)_{n-m} .
\end{aligned}
$$

Now, proceeding exactly as before, taking account of (2.11) and the further Hankel transform given by [13]

$$
\begin{aligned}
\int_{0}^{\infty} x^{-\kappa-1} \log x J_{2 \sigma}(2 \sqrt{x y}) \mathrm{d} x \\
=y^{\kappa} \Gamma(\sigma-\kappa) / \Gamma(\sigma+\kappa+1)[\psi(\sigma-\kappa)+\psi(\sigma+\kappa+1)-\log y] \\
(0 \leqslant \kappa \leqslant \sigma)
\end{aligned}
$$

we deduce that

$$
\begin{aligned}
\int_{0}^{\infty} \mathrm{e}^{-x} x^{\sigma-\kappa-1} & \log x L_{p}^{\sigma+q-p}(x) L_{q}^{\sigma+p-q}(x) \mathrm{d} x \\
= & (-1)^{p+q} \Gamma(\sigma-\kappa) /\left[\Gamma(\sigma+\kappa+1)(\kappa+1)_{p-q}(\kappa+1)_{q-p}\right] \\
& \times([\psi(\sigma-\kappa)+\psi(\sigma+\kappa+1)-2 \psi(\kappa+1) \\
& +\psi(\kappa+1+p-q)+\psi(\kappa+1+q-p)] \\
& \times \int_{0}^{\infty} \mathrm{e}^{-x} x^{\sigma+\kappa} L_{p}^{\sigma+q-p}(x) L_{q}^{\sigma+p-q}(x) \mathrm{d} x \\
& \left.-\int_{0}^{\infty} \mathrm{e}^{-x} x^{\sigma+\kappa} \log x L_{p}^{\sigma+q-p}(x) L_{q}^{\sigma+p-q}(x) \mathrm{d} x\right)
\end{aligned}
$$

for $0 \leqslant \kappa \leqslant \sigma$. When $\kappa$ is a non-negative integer less than $|p-q|, 1 /(\kappa+1)_{-|p-q|}$ is zero, but then so is $1 / \psi(\kappa+1-|p-q|)$. Thus in this latter case only one term on the RHS of $(3.5)$ is non-zero; in fact

$$
\begin{aligned}
\int_{0}^{\infty} \mathrm{e}^{-x} x^{\sigma-\kappa-1} & \log x L_{p}^{\sigma+q-p}(x) L_{q}^{\sigma+p-q}(x) \mathrm{d} x \\
= & (-1)^{p+q} \Gamma(\sigma-\kappa) /\left[\Gamma(\sigma+\kappa+1)(\kappa+1)_{p-q}\right] \\
& \times \int_{0}^{\infty} \mathrm{e}^{-x} x^{\sigma+\kappa} L_{p}^{\sigma+q-p}(x) L_{q}^{\sigma+p-q}(x) \mathrm{d} x
\end{aligned}
$$

It is now a simple matter to deduce the formulae relating matrix elements of $r^{k}$ and $r^{k} \log r$ presented by Blanchard [1]. We find, for $k=0,1, \ldots,\left|l-l^{\prime}\right|-1$ $\left\langle n l^{\prime}\left|r^{-k-2} \log 2 r / a n\right| n l\right\rangle$

$$
=(-1)^{k}(2 / a n)^{k} \frac{\left(l+l^{\prime}-k\right) !\left(\left|l-l^{\prime}\right|-k-1\right) ! k !^{2}}{\left(l+l^{\prime}+k+1\right) !\left(k+\left|l-l^{\prime}\right|\right) !}\left\langle n l^{\prime}\left|r^{k-1}\right| n l\right\rangle
$$

and for $0 \leqslant \kappa \leqslant l+l^{\prime}$ 
$\left\langle n l^{\prime}\left|r^{-\kappa-2} \log 2 r / a n\right| n l\right\rangle$

$$
\begin{aligned}
= & (-1)^{l+l^{\prime}}(2 / a n)^{2 \kappa+1} \frac{\Gamma\left(l+l^{\prime}+1-\kappa\right) \Gamma(\kappa+1)^{2}}{\Gamma\left(l+l^{\prime}+\kappa+2\right) \Gamma\left(\kappa+l-l^{\prime}+1\right) \Gamma\left(\kappa+l^{\prime}-l+1\right)} \\
& \times\left\{\left[\psi\left(l+l^{\prime}+1-\kappa\right)+\psi\left(l+l^{\prime}+\kappa+2\right)-2 \psi(\kappa+1)+\psi\left(\kappa+l-l^{\prime}+1\right)\right.\right. \\
& \left.\left.+\psi\left(\kappa+l^{\prime}-l+1\right)\right]\left\langle n l^{\prime}\left|r^{\kappa-1}\right| n l\right\rangle-\left\langle n l^{\prime}\right| r^{\kappa-1} \log 2 r / \text { an }|n l\rangle\right\}
\end{aligned}
$$

which are the results established by Blanchard [1].

In summary, the derivation presented here reveals that equations (2.8) and (2.9) are the fundamental integral properties of the Laguerre polynomials which, together with Erdelyi's relation (2.11), leads to the identity (1.1) for the radial matrix elements. In the case of integrals involving $\log x$, the corresponding integral property is equation (3.3).

\section{Acknowledgments}

Research support by the Natural Sciences and Engineering Research Council of Canada is gratefully acknowledged.

\section{References}

[1] Blanchard P 1974 J. Phys. B: At. Mol. Phys. 7993

[2] Bethe H A and Salpeter E E 1957 Quantum Mechanics of One-and Two-Electron Atoms (New York: Academic)

[3] Lebedev N N 1972 Special Functions and their Applications (New York: Dover)

[4] Pasternak S 1937 Proc. Natl. Acad. Sci. 2391

[5] Pasternak S and Sternheimer R M 1962 J. Math. Phys. 31280

[6] More R M 1983 J. Phys. B: At. Mol. Phys. 163859

[7] Ojha P C and Crothers D S F 1984 J. Phys. B: At. Mol. Phys. 174797

[8] Armstrong L L $1970 \mathrm{~J}$. Physique $31 \mathrm{C} 417$

[9] Ding Y B 1987 J. Phys. A: Math. Gen. 206293

[10] Khandelwal G S, Pritchard W M, Grubb G, Khan F 1989 Phys. Rev. A 393960

[11] Bockasten K 1974 Phys. Rev. A 91087

[12] Badawi M, Bessis N, Bessis G, Hadinger G 1973 Phys. Rev. A 8727

[13] Gradshteyn I S and Ryzhik I M 1980 Table of Integrals, Series and Products (New York: Academic)

[14] Erdelyi A 1938 J. London Math. Soc. 13146 